OPEN ACCESS

Edited by:

Sven Haller,

Rive Droite SA, Switzerland

Reviewed by:

Gianluca Serafini,

San Martino Hospital (IRCCS), Italy

Luke Norman,

University of Michigan,

United States

*Correspondence:

Linyan Su

xysulinyan@126.com

Qing Jiao

bingbao17@163.com

Specialty section:

This article was submitted to Neuroimaging and Stimulation,

a section of the journal

Frontiers in Psychiatry

Received: 27 February 2020

Accepted: 12 June 2020

Published: 26 June 2020

Citation:

Cui D, Guo Y, Cao W, Gao W, Qiu J, Su L, Jiao $Q$ and Lu G (2020) Correlation Between Decreased

Amygdala Subnuclei Volumes and Impaired Cognitive Functions in Pediatric Bipolar Disorder.

Front. Psychiatry 11:612. doi: 10.3389/fpsyt.2020.00612

\section{Correlation Between Decreased Amygdala Subnuclei Volumes and Impaired Cognitive Functions in Pediatric Bipolar Disorder}

\author{
Dong Cui ${ }^{1,2}$, Yongxin Guo ${ }^{1,2}$, Weifang Cao ${ }^{1,2}$, Weijia $\mathrm{Gao}^{3}$, Jianfeng $\mathrm{Qiu}^{1}$, Linyan $\mathrm{Su}^{4 *}$, \\ Qing Jiao ${ }^{1,2 *}$ and Guangming $\mathrm{Lu}^{5}$ \\ ${ }^{1}$ College of Radiology, Shandong First Medical University (Shandong Academy of Medical Sciences), Taian, China, \\ ${ }^{2}$ Collaborative Innovation Center of Magnetic Resonance Imaging of Brain Disease, Shandong First Medical University, \\ Shandong Academy of MedicalSciences, Taian, China, ${ }^{3}$ Department of Child Psychology, The Children's Hospital, Zhejiang \\ University School of Medicine, Hangzhou, China, ${ }^{4}$ Mental Health Institute of The Second Xiangya Hospital, Central South \\ University, Changsha, China, ${ }^{5}$ Department of Medical Imaging, Jinling Hospital, Nanjing University School of Medicine, \\ Nanjing, China
}

Background: The amygdala has been proposed to be involved in the pathophysiology of pediatric and adult bipolar disorder (BD). The goal of this structural magnetic resonance imaging (sMRI) study was to investigate the morphometric characteristics of amygdala subnuclei in patients with pediatric bipolar disorder (PBD) compared to healthy controls (HCs). Simultaneously, we examined correlation between amygdala subnuclei volumes and cognitive dysfunction.

Materials and Methods: We assessed 40 adolescent outpatients, diagnosed with manic or euthymic PBD according to the DSM-5 criteria for BD and $19 \mathrm{HCs}$. Cognitive functions were evaluated using a Stroop color-word test (SCWT), trail making test (TMT), visual reproduction immediate recall subtest (VR I), and digit span subtest (DST). Amygdala and its subnuclei structures were automated segmented using FreeSurfer software and the volumes of them were compared between groups and correlation with clinical and cognitive outcomes was conducted.

Results: Manic patients exhibited significantly decreased volumes in the bilateral whole amygdala and its basal nucleus, cortico-amygdaloid transition (CAT), and accessory basal nucleus (ABN) compared with HCs. Euthymic patients had decreased volume in the bilateral $\mathrm{ABN}$ and left CAT. In addition, we found significant positive associations between VR I scores and the right whole amygdala and its bilateral basal, right lateral, and $A B N$ volumes in the manic group.

Conclusion: These findings support previous reports of smaller amygdala volumes and cognitive dysfunctions in PBD, and further mapping abnormalities to specific amygdala subnuclei. Correlation between basolateral volume and VR I of PBD may expand our understanding of neural abnormalities that could be targeted by treatment.

Keywords: pediatric bipolar disorder, mania, euthymia, amygdala subnuclei, magnetic resonance imaging 


\section{INTRODUCTION}

Pediatric bipolar disorder (PBD) is characterized by persistent influence dysregulation affects roughly $2 \%$ of youth under the age of 18 (1). Like bipolar disorder (BD) in adults, PBD is also characterized by recurring manic or hypomanic episodes and a depressive episode typically separated by periods of relative euthymia (2). Retrospective studies clearly indicate that pathology begins in childhood or adolescence for $50 \%$ to $66 \%$ of adults with BD (3). Early-onset BD may have worse outcomes including greater cognitive impairment (4), fewer days of euthymia (5), and suicide attempts (6). In these adolescents, the persistent affect dysregulation is often accompanied by increased risk of suicide (7) and severe cognitive impairment (8) leading to considerable deficits in memory, executive, processing speed, and verbal learning $(5,9)$. Therefore, it is important to have early objective biomarkers to detect cognitive impairment in order to minimize its negative impact on adolescent development. Such a biomarker would allow early and reliable identification and treatment of $\mathrm{BD}$ disorderassociated cognitive decline and shed light on the underlying mechanisms of $\mathrm{BD}$ development. However, no biomarkers for targeting or tracking the progression of $\mathrm{BD}$ in adolescents exist.

The amygdala is a key limbic region in modulating mood and emotions and is potentially involved in the cognitive and affective symptoms of BD (10). Neuropathologic and neuroimaging studies have implicated the amygdala as a central brain structure for processing emotions $(11,12)$, emotion-related aspects of behavior (13), attention (14), and memory (15). Converging evidence from neuroimaging studies has consistently implicated the dysfunction of the amygdala in the pathophysiology of BD. Kryza-Lacombe and colleagues (16) showed that youth and adult patients with BD had abnormal amygdala-temporo-parietal connectivity. Specifically, amygdala activation is inversely correlated with volume (17).

Numerous structural magnetic resonance imaging (MRI) studies indicate that smaller amygdala volumes may be an agespecific biomarker for BD. Decreased amygdala volumes in patients with $\mathrm{PBD}$ as compared with HCs have been reported in most studies (18-21). In contrast, studies of adults BD patients regarding the amygdala are markedly heterogeneous, with increased (22), not significantly different $(23,24)$, or decreased (25-27) amygdala volumes compared with HCs. These discrepancies likely reflect clinical and treatment heterogeneity. Some researchers speculate that amygdala volume is reduced at the onset of the disease and increases with age (26). A metaanalysis of the functional neural correlates of $\mathrm{BD}$ highlighted the amygdala as an area with unique developmental alterations in BD (28). Therefore, structural and functional amygdala abnormalities identified by neuroimaging may serve as useful disease and treatment response biomarker in $\mathrm{BD}$.

The amygdala formation is commonly treated as a single entity in structural MRI; however, it is known to be comprised of multiple nuclei, each exhibiting different connectivities and cellular profiles (29). These subnuclei have diverse functions physiologically and have been shown in disease models of $\mathrm{BD}$ to react differentially to pathological mechanisms $(30,31)$. Due to the small size of the amygdala, few studies focused on volume changes of amygdala subnuclei in patients with PBD. Whether smaller amygdala volume has been localized to specific amygdala subnuclei in different clinical stages is unknown. With substantial advances in structural MRI tools, new amygdala segmentation algorithms have made it possible to label amygdala subnuclei and automatically provide volumetric information for each based on an in vivo atlas (32). Given this background, the goal of the current study was to compare amygdala and subnuclei volumes in a sample of manic or euthymic patients with PBD, and HCs. We hypothesized that the volumes of amygdala subnuclei would be smaller in patients with PBD than that of HCs. Moreover, we also hypothesized that worse cognitive abnormalities might be associated with these reduced amygdala subnuclei in patients with mania or euthymia.

\section{MATERIALS AND METHODS}

\section{Subjects}

In this case-control study, all PBD patients were recruited from the Mental Health Institute of the Second Xiangya Hospital, Key Laboratory of Psychiatry and the Mental Health of Hunan Province of Central South University (Changsha, Hunan, China). We recruited forty right-handed patients with $\mathrm{PBD}$ across an age range of 12 to 18 years. All patients met DSM-5 criteria for BD (33), made up of two subgroups, mania $(n=20,9$ male/11 female), and euthymia ( $\mathrm{n}=20,11 \mathrm{male} / 9$ female). In addition, 19 right-handed age and sex-matched healthy control (HC) participants ( 7 male/12 female) were recruited from the local middle school via advertisements. All subjects were completed the Wechsler Abbreviated Scale of Intelligence as an overall measure of cognitive ability (34). General exclusion criteria were intellectual disability (IQ $\leq 80$ ), left-handedness, substance abuse, history of seizures, history of electroconvulsive therapy (ECT), severe brain trauma, and MRI scan contraindications (e.g. metallic implants or claustrophobia).

All adolescents were assessed by the Schedule for Affective Disorders and Schizophrenia for School-Age Children-Present and Lifetime (KSADS-PL) (35) and the Washington University in St. Louis Kiddie Schedule for Affective Disorders and Schizophrenia (WASH-U-KSADS) (36). The K-SADS-PL for DSM-5 is a semi-structured diagnostic interview that assesses both current and lifetime diagnostic psychiatric episodes in children and adolescents. WASH-U-KSADS was developed specifically to target the assessment of prepubertal mania and hypomania and to assess the pattern of rapid cycling. Furthermore, severity of depression and mania were evaluated in all subjects by the Mood and Feelings Questionnaire (MFQ) (37), and Young Manic Rating Scale (YMRS) (38) respectively. The MFQ is a widely used screening measure of depressive symptomatology for children 8 to 18 years of age. The YMRS is an instrument used to assess the severity of mania in patients with a diagnosis of $\mathrm{BD}$. The patients in the manic subgroup were required to have a YMRS score $>26$ and MFQ score $<18$, those in the euthymic subgroup were required to have had no episodes 
of illness for at least 1 month and YMRS score $<12$ and MFQ score $<18$ at the time of scanning. The inclusion criteria for $\mathrm{HC}$ included that the participants have no current or past DSM-5 psychiatric diagnosis, as confirmed by KSADS-PL, and no first- or second-degree family history of $\mathrm{BD}$ or other psychotic disorders.

This study protocol was approved by the University of Central South Institutional Review Board in compliance with the Declaration of Helsinki. After complete description of the study to adolescents and their parents, written informed consent and assent were obtained.

\section{Cognitive Function Test}

To assess different aspects of cognitive functions, the cognitive estimate battery included the following: Stroop color-word test (SCWT), trail making test (TMT), visual reproduction immediate recall subtest (VR I), and digit span subtest (DST). The battery was administered by experienced clinical psychiatrists in a quiet environment. Below is a description of the various test procedures.

\section{Stroop Color-word Test (SCWT)}

The SCWT (39), measuring the ability to attention and response inhibition, included three tasks: word reading (SCWT-A), color naming (SCWT-B), and color interference reading (SCWT-C), each set contains 100 visual stimuli. SCWT-A is made up of the number of words that participants completed in $45 \mathrm{~s}$. SCWT-B is made up of the number of symbols that subjects named correctly. SCWT-C is made up of the number of competing colors that participants read in $45 \mathrm{~s}$.

\section{Trail Making Test (TMT)}

TMT is administered in the part A (TMT-A) and part B (TMTB). TMT-A requires the subjects to draw a line between consecutive numbers (1-25) distributed on a piece of paper, and TMT-B requires the subjects to draw lines sequentially connecting 13 numbers (1-13) and 12 letters (A-L) distributed on a piece of paper. Numbers and letters are encircled and must be connected alternately. TMT score was the total times for subjects to complete the task. TMT-A reflect attention and processing speed, and part B reflects cognitive flexibility (40).

\section{Visual Reproduction Immediate Recall Subtest (VR I)}

The VR I was used to assessing visual memory, which check immediate recall and learning rate. Three pages of geometric designs are shown, one at a time. After viewing each graphic for $10 \mathrm{~s}$, the participants are instructed to draw the graphics as accurately as possible from memory (41).

\section{Digit Span Subtest (DST)}

In the DST, the participant is asked to repeat the same sequence numbers back to the psychiatrists in forward order (DST-A) and in reverse order (DST-B). DST-A and DST-B were scored according to the longest series separately. DST-A was used to assess attention and DST-B measured working memory (34).

\section{MRI Acquisition and Analysis}

All MRI scans were collected with a 3.0 T Siemens Trio system (Siemens, Erlangen, German) using a standard whole head coil. High-resolution anatomical scan was acquired using threedimensional magnetization-prepared rapid acquisition gradient echo (3D MPRAGE) protocol with the following parameters: repetition time $(\mathrm{TR})=2300 \mathrm{~ms}$, echo time $(\mathrm{TE})=2.98 \mathrm{~ms}$, inversion time $=900 \mathrm{~ms}$, thickness $=1 \mathrm{~mm}$, gap $=0 \mathrm{~mm}$, field of view $($ FOV $)=256 \mathrm{~mm} \times 256 \mathrm{~mm}$, matrix $=256 \times 256$, flip angle $=9^{\circ}$.

T1-weighted images were preprocessed by motion correction and brain extraction using FreeSurfer (version 6.0, https://surfer. nmr.mgh.harvard.edu). Each T1-weighted image was segmented into gray matter (GM), white matter (WM), and cerebrospinal fluid (CSF). Subsequently, the segmentation of subcortical structures was examined by a nonlinear warping atlas, yielding volumetric measures of Deep GM, including the thalamus, caudate, putamen, amygdala, hippocampus, pallidum, and accumbens. Furthermore, the amygdala subnuclei segmentation module, which is only present in the FreeSurfer dev version (ftp://surfer.nmr.mgh.harvard.edu/pub/dist/freesurfer/dev) was used to parcellate the hippocampus, amygdala, and thalamus subnuclei further, as shown in Figure 1A. A probabilistic atlas and a modified version of Van Leemput's algorithm was applied on the segmentation of amygdala (32). In total the amygdala was divided into nine nuclei, including lateral, basal, accessory-basal nucleus (ABN), anterior-amygdaloid area (AAA), central, medial, cortical, cortico-amygdaloid transition (CAT), and paralaminar nucleus. Finally, using FreeSurfer's native visualization toolbox, freeview, we visually inspected the segmentation of hippocampal/amygdala, as shown in Figure 1.

We used the Statistical Package for the Social Sciences (SPSS) for Windows, version 22.0 (SPSS Statistics, IBM, Armonk, NY, USA) to study the demographic, clinical, cognitive tests, and MRI data. The Shapiro-Wilk test was used to test for normality. Demographic, clinical, and cognitive test scores were evaluated using Pearson's chisquare test, two-sample t-test or one-way ANOVA with a confidence interval of $95 \%$ where applicable. A general linear model (GLM) was used for group analysis of each subnuclei. The GLM was fitted with volume as the dependent variable, groups as the categorical predictor, and total intracranial volume (TIV), age, gender, and education were included as covariates. The indices with significant differences across the three groups were examined further by post-hoc differences. Multiple comparisons between groups were assessed using the Bonferroni method. We calculated the Spearman correlation coefficients between each subregion volume and each of the clinical and cognitive variables (onset age, illness duration, YMRS scores, and cognitive tests) for the PBD patients. In the correlation calculations, we regressed out the confounding factors of age, gender, education, and TIV. Spearman correlation results were corrected by false discovery rate (FDR) correction. Statistical significance for all tests was set at $p<0.05$. 


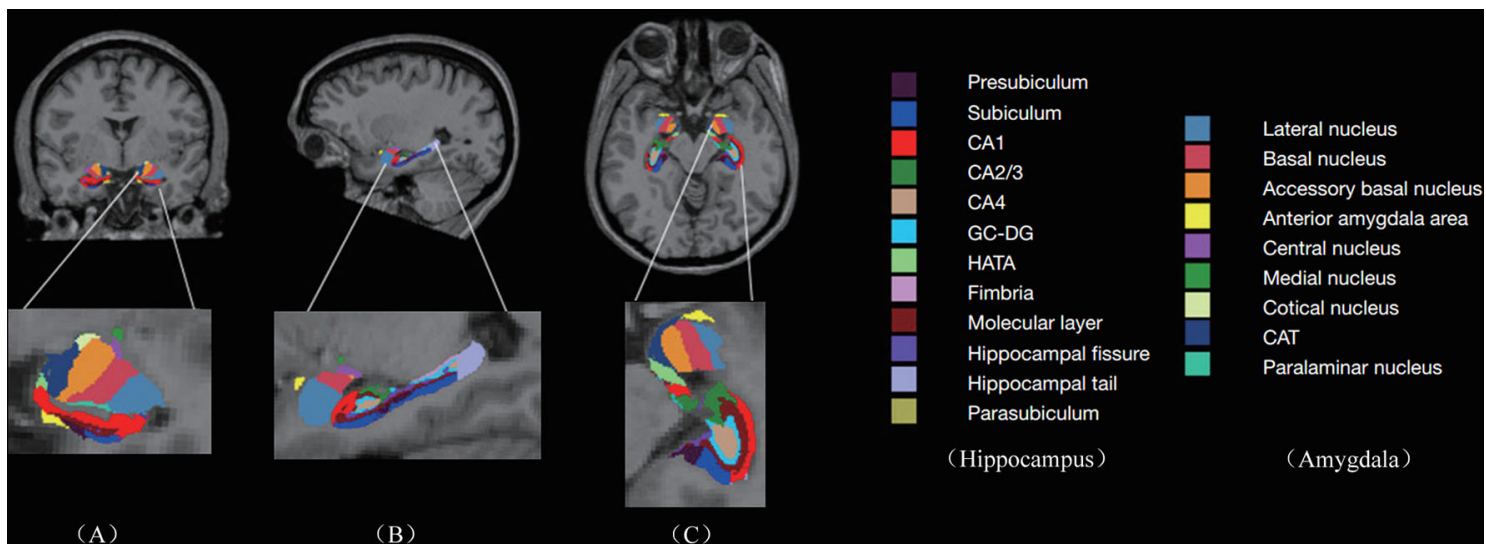

FIGURE 1 | Subregions of hippocampus and amygdala: Columns (A-C) represent the image view of coronal, sagittal, and axial, respectively. The second row represents the enlarged subregions on the left. CA, cornus ammonis; GC-DG, granule cell layer of the dentate gyrus; HATA, hippocampal amygdala transition area; CAT, cortico-amygdaloid transition.

\section{RESULTS}

\section{Participants' Characteristics and Cognitive Tests}

Clinical, demographic, and cognitive test information was collected through self-report questionnaires and clinical interviews by trained psychiatrists. Demographic, clinical, cognitive tests, and medication regimen are summarized in Table 1. No group differences were observed in age $(F=3.118$, $p=0.052)$, gender (chi-square $=1.301, p=0.522)$, education $(F=$ $2.153, \mathrm{p}=0.126)$, IQ $(F=1.503, p=0.231)$ or MFQ $(F=0.429$, $p=0.654)$. As expected, significant group differences were observed for YMRS $(F=356.537, p<0.001)$. There were no significant differences in age of onset $(\mathrm{t}=0.587, p=0.561)$, illness duration $(\mathrm{t}=-1.687, p=0.100)$, onset frequency $(\mathrm{t}=-0.983, p=$ 0.332 ), psychotic symptoms (chi-square $=0.902, p=0.342$ ), type of $\mathrm{BD}$ (chi-square $=0.125, p=0.723$ ) or familial BD history (chisquare $=0.476, p=0.490$ ) between two groups of PBD patients. In the mania subgroup, patients were taking the following medications: lithium $(\mathrm{n}=8)$, valproate $(\mathrm{n}=11)$, antipsychotics $(\mathrm{n}=13)$, and antidepressants $(\mathrm{n}=2)$. In the euthymic subgroup, patients were taking lithium $(\mathrm{n}=8)$, valproate $(\mathrm{n}=15)$, and antipsychotics $(\mathrm{n}=15)$. There were significant differences in SCWT-A scores $(F=4.852, p=0.011)$, SCWT-B scores $(F=$ $8.023, p=0.001)$, SCWT-C scores $(F=9.161, p<0.001)$, TMT-A scores $(F=4.439, p=0.016)$, VR I scores $(F=16.132, p<0.001)$, and DST-B scores $(F=5.412, p=0.007)$. Furthermore, the pairwise comparisons demonstrated apparent declines in SCWT, VR I, and DST-B scores in the two PBD groups $(p<0.05)$ compared with the HC group, as well as lower TMT-A scores in the manic patients $(p<0.05)$ compared with HC (Table 1). No significant difference was observed for TMT-B scores $(F=1.455$, $p=0.242)$ or DST-A scores $(F=1.520, p=0.228)$ among the 3 groups.

\section{Subnuclei Volume Analysis}

Table 2 summarizes the statistical analysis for the volume of the amygdala subnuclei. There were significant differences in the bilateral whole amygdala, basal nucleus, ABN, and CAT, left cortical nucleus, left paralaminar nucleus, and right central nucleus among the three groups $(p<0.05)$. Histograms in Figure 2 demonstrate post-hoc pairwise comparisons on amygdala subnuclei volumes. Table 2 lists the statistical results of post-hoc pairwise comparisons in subnuclei with significant differences among the three groups. The strongest effects for bilateral whole amygdala, $\mathrm{ABN}$, and $\mathrm{CAT}$ volume decrease were seen in manic patients $(p<0.01)$. In addition, euthymic PBD group exhibited deceased bilateral $\mathrm{ABN}$ and left CAT volumes compared with HCs $(p<0.05)$.

\section{Correlation Analysis}

Age, gender, and years of education were not significantly correlated with amygdala morphology within the HCs and PBD groups. In the manic PBD group, VR I score was found to be positively correlated with right whole amygdala, bilateral basal nucleus, right lateral nucleus, and right $\mathrm{ABN}$ volume $(p<$ 0.05 ; Figure 3). The euthymic PBD group had no significant correlation between the subnuclei volume and any of the clinical and cognitive characteristics.

\section{DISCUSSION}

To the best of our knowledge, this cross-sectional study is the first work utilizing automated neuroanatomical quantification (FreeSurfer) to evaluate amygdala and subnuclei volumetric differences in PBD patients. The main finding of the present study was the significant differences in the basal nucleus, $A B N$, and CAT volumes between PBD patients and HCs. Unexpectedly, 
TABLE 1 | Sample characteristics.

\begin{tabular}{|c|c|c|c|c|c|c|c|c|c|}
\hline \multirow{2}{*}{\multicolumn{2}{|c|}{ Characteristics }} & \multirow{2}{*}{$\begin{array}{l}\text { Manic-PBD } \\
\qquad(n=20)\end{array}$} & \multirow{2}{*}{$\begin{array}{l}\text { Euthymic-PBD } \\
\qquad(n=20)\end{array}$} & \multirow[t]{2}{*}{$H C(n=19)$} & \multirow[t]{2}{*}{$F / T / \chi^{2}$} & \multirow[t]{2}{*}{$p$} & \multicolumn{3}{|c|}{ Pairwise comparisons ( $p$ value) } \\
\hline & & & & & & & $\begin{array}{l}\text { Mania vs. } \\
\text { Euthymia }\end{array}$ & $\begin{array}{l}\text { Mania vs. } \\
\text { HC }\end{array}$ & $\begin{array}{l}\text { Euthymia vs. } \\
\qquad \text { HC }\end{array}$ \\
\hline \multicolumn{2}{|c|}{ Gender (male/female) } & $9 / 11$ & $11 / 9$ & $7 / 12$ & $1.301^{\#}$ & 0.522 & 0.527 & 0.605 & 0.256 \\
\hline \multicolumn{2}{|l|}{ Age (years) } & $15.30 \pm 1.81$ & $15.60 \pm 1.64$ & $14.37 \pm 1.30$ & $3.118^{\&}$ & 0.052 & 1.000 & 0.059 & 0.224 \\
\hline \multicolumn{2}{|c|}{ Education (years) } & $8.40 \pm 1.76$ & $8.70 \pm 1.75$ & $7.47 \pm 2.22$ & $2.153^{\&}$ & 0.126 & 1.000 & 0.411 & 0.152 \\
\hline \multicolumn{2}{|c|}{$\mathrm{IQ}$} & $103.50 \pm 10.67$ & $108.60 \pm 9.73$ & $105.32 \pm 7.51$ & $1.503^{\&}$ & 0.231 & 0.278 & 1.000 & 0.844 \\
\hline \multicolumn{2}{|c|}{ YMRS scores } & $34.30 \pm 6.44$ & $5.50 \pm 1.70$ & $3.63 \pm 2.06$ & $356.537^{\&}$ & $<0.001^{\star \star \star}$ & $<0.001^{\star \star \star}$ & $<0.001^{\star \star \star}$ & 0.467 \\
\hline \multicolumn{2}{|c|}{ MFQ scores } & $7.15 \pm 2.62$ & $6.65 \pm 4.38$ & $6.11 \pm 3.33$ & $0.429^{\&}$ & 0.654 & 1.000 & 1.000 & 1.000 \\
\hline \multicolumn{2}{|c|}{ Onset age (year) } & $14.05 \pm 1.73$ & $13.70 \pm 2.03$ & - & $0.587^{\wedge}$ & 0.561 & - & - & - \\
\hline \multicolumn{2}{|c|}{ Illness duration (months) } & $15.90 \pm 12.96$ & $24.15 \pm 17.62$ & - & $-1.687^{\wedge}$ & 0.100 & - & - & - \\
\hline \multicolumn{2}{|c|}{ Onset frequency } & $3.10 \pm 1.68$ & $4.80 \pm 7.55$ & - & $-0.983^{\wedge}$ & 0.332 & - & - & - \\
\hline \multicolumn{2}{|c|}{$\begin{array}{l}\text { The first episode bipolar disorder } \\
\text { (mania/depression) }\end{array}$} & $9 / 11$ & $7 / 13$ & - & $0.417^{\#}$ & 0.519 & - & - & - \\
\hline \multicolumn{2}{|c|}{$\begin{array}{l}\text { Acute or delayed onset (acute/ } \\
\text { delayed) }\end{array}$} & $10 / 10$ & $11 / 9$ & - & $0.010^{\#}$ & 0.752 & - & - & - \\
\hline \multicolumn{2}{|c|}{ Psychotic symptoms(yes/no) } & $9 / 11$ & $12 / 8$ & - & $0.902^{\#}$ & 0.342 & - & - & - \\
\hline \multicolumn{2}{|c|}{ BP-I/BP-II } & $15 / 5$ & $14 / 6$ & - & $0.125^{\#}$ & 0.723 & - & - & - \\
\hline \multicolumn{2}{|c|}{ Familial BD history(yes/no) } & $7 / 13$ & $5 / 15$ & - & $0.476^{\#}$ & 0.490 & - & - & - \\
\hline \multirow[t]{4}{*}{ Medications } & Lithium & 8 & 8 & - & - & - & - & - & \\
\hline & Valproate & 11 & 13 & - & - & - & - & - & \\
\hline & $\begin{array}{l}\text { Atypical } \\
\text { antipsychotics }\end{array}$ & 13 & 15 & - & - & - & - & - & \\
\hline & Antidepressants & 2 & - & - & - & - & - & - & \\
\hline \multicolumn{2}{|l|}{ SCWT-A } & $53.35 \pm 15.98$ & $54.35 \pm 13.53$ & $66.00 \pm 12.26$ & $4.852^{\&}$ & $0.011^{*}$ & 1.000 & $0.020^{\star}$ & $0.037^{\star}$ \\
\hline \multicolumn{2}{|l|}{ SCWT-B } & $69.80 \pm 19.56$ & $71.80 \pm 15.16$ & $87.79 \pm 9.08$ & $8.023^{\&}$ & $0.001^{\star \star}$ & 1.000 & $0.002^{\star \star}$ & $0.006^{\star \star}$ \\
\hline \multicolumn{2}{|l|}{ SCWT-C } & $29.65 \pm 7.36$ & $31.85 \pm 9.12$ & $40.74 \pm 9.43$ & $9.161^{\&}$ & $<0.001^{\star \star \star}$ & 1.000 & $<0.001^{\star \star \star}$ & $0.006^{\star \star}$ \\
\hline \multicolumn{2}{|l|}{ TMT-A } & $40.35 \pm 12.31$ & $38.15 \pm 12.80$ & $29.74 \pm 9.63$ & $4.439^{\&}$ & $0.016^{\star}$ & 1.000 & $0.019^{\star}$ & 0.086 \\
\hline \multicolumn{2}{|l|}{ TMT-B } & $88.90 \pm 30.64$ & $101.95 \pm 50.89$ & $80.61 \pm 31.27$ & $1.455^{\&}$ & 0.242 & 0.844 & 1.000 & 0.294 \\
\hline \multicolumn{2}{|l|}{ VRT } & $8.45 \pm 3.41$ & $10.30 \pm 2.60$ & $13.21 \pm 1.48$ & $16.132^{\&}$ & $<0.001^{\star \star \star}$ & 0.091 & $<0.001^{\star \star \star}$ & $0.003^{\star \star}$ \\
\hline \multicolumn{2}{|l|}{ DST-A } & $8.20 \pm 1.51$ & $8.65 \pm 1.66$ & $9.00 \pm 1.05$ & $1.520^{\&}$ & 0.228 & 0.980 & 0.264 & 1.000 \\
\hline \multicolumn{2}{|l|}{ DST-B } & $4.45 \pm 1.15$ & $4.75 \pm 1.62$ & $5.95 \pm 1.68$ & $5.412^{\&}$ & $0.007^{\star \star}$ & 1.000 & $0.009^{\star \star}$ & $0.047^{\star}$ \\
\hline
\end{tabular}

Data are presented as mean \pm standard deviation. ${ }^{\#}$ Pearson chi-square test; ${ }^{\circledR}$ one-way ANOVA (analysis of variance); ${ }^{\wedge}$ Independent-sample t-test. The pairwise comparisons between groups using Bonferroni method. ${ }^{*} p<0.05,{ }^{* *} p<0.01,{ }^{* * *} p<0.001$.

IQ, intelligence quotient; YMRS, Young Manic Rating Scale; MFQ, Mood and Feelings Questionnaire; BP-I, bipolar disorder type I; BP-II, bipolar disorder type II; SCWT, Stroop color-word test; TMT, trail making test; VR I, visual reproduction immediate recall subtest; DST, digit span test.

amygdala and subnuclei volumes between manic and euthymic patient group were indistinguishable for all structures examined. In addition, this study detected that PBD patients have significant differences in SCWT, TMT-A, VR I, and DST-B compared to HCs. And in the manic PBD group, VR I score was found to be positively correlated with right whole amygdala, bilateral basal nucleus, right lateral nucleus, and right $\mathrm{ABN}$ volume.

Neuroimaging studies in PBD have so far supported the key role of amygdala. Alterations in amygdala volumes have been associated with measures of illness duration and disease progression in PBD (20). Consistent with previous finding, the present study specifically found a mean volume reduction of $6.4 \%$ in left amygdala, and $7.8 \%$ in right amygdala in manic PBD patients compared with that of the HCs, with no significant difference in euthymic PBD patients (Figure 2A). Furthermore, the mania and euthymia group showed differences in right whole amygdala volume, but it did not attain the statistical significance $(p=0.050)$. It is worth noting that little evidence of amygdala volumetric alterations was reported in young subjects with schizophrenia (SZ) or other psychotic disorders, indicating that alterations may be specific to BD (42). Post mortem studies had reported the amygdala as a common site for senile plaques and neurofibrillary tangles in Alzheimer's disease (AD) and mild cognitive impairment (43). McGaugh emphasized that the amygdala is critically related with memory consolidation by intermediating the impacts of epinephrine and glucocorticoids and regulating the activities of striatum and hippocampus (44). In healthy individuals, amygdala volume has no connection with memory function, whereas in BD patients, larger amygdala volume was predictive of integrated memory function (24). Consistent with our study, we found that right whole amygdala volume was predictive of cognitive performance in manic group, correlating positively with better immediate recall memory for manic PBD patients (Figure 3A).

We determined in vivo localization of the volumetric difference within the amygdala. The most affected subnuclei were the bilateral $\mathrm{ABN}$ and left CAT in all $\mathrm{PBD}$ patients (Figures 2D, I), and volume changes in the bilateral basal, left paralaminar nucleus, and right CAT, lateral, and central nucleus were apparent only in manic patients (Figures 2B, C, I, J). Originating in the anteromedial temporal lobe, the amygdalofugal tract passes through the basal, lateral, and central amygdala nucleus toward the midline (45), which is believed to apply downstream control over hypothalamus and septal nuclei, 
TABLE 2 | The difference among the three groups in the amygdala and subnuclei.

\begin{tabular}{|c|c|c|c|c|c|c|c|c|c|}
\hline \multicolumn{2}{|c|}{ Regions } & \multirow[t]{2}{*}{ Manic-PBD $(n=20)$} & \multirow{2}{*}{$\begin{array}{l}\text { Euthymic-PBD } \\
\qquad(\mathrm{n}=20)\end{array}$} & \multirow[t]{2}{*}{$H C(n=19)$} & \multirow[t]{2}{*}{$F^{\#}$} & \multirow[t]{2}{*}{$p$} & \multicolumn{3}{|c|}{ Pairwise comparisons ( $p$ value) } \\
\hline & & & & & & & $\begin{array}{l}\text { Mania vs. } \\
\text { Euthymia }\end{array}$ & $\begin{array}{c}\text { Mania vs. } \\
\text { HC }\end{array}$ & $\begin{array}{c}\text { Euthymia vs. } \\
\text { HC }\end{array}$ \\
\hline \multirow[t]{10}{*}{ Left } & Whole amygdala & $1699.614 \pm 193.038$ & $1805.293 \pm 195.487$ & $1815.260 \pm 174.344$ & 4.669 & $0.014^{\star}$ & 0.145 & $0.004^{\star \star}$ & 0.121 \\
\hline & Lateral nucleus & $647.823 \pm 72.738$ & $683.317 \pm 70.861$ & $676.520 \pm 65.766$ & 1.776 & 0.180 & 0.274 & 0.068 & 0.447 \\
\hline & Basal nucleus & $439.095 \pm 54.010$ & $464.978 \pm 55.652$ & $468.220 \pm 47.291$ & 3.274 & $0.046^{\star}$ & 0.263 & $0.013^{\star}$ & 0.161 \\
\hline & $\mathrm{ABN}$ & $250.118 \pm 27.183$ & $269.462 \pm 32.278$ & $275.435 \pm 31.274$ & 8.345 & $0.001^{\star *}$ & 0.075 & $<0.001^{\star \star \star}$ & $0.028^{*}$ \\
\hline & AAA & $58.851 \pm 8.472$ & $60.574 \pm 7.341$ & $60.381 \pm 6.779$ & 0.113 & 0.893 & 0.901 & 0.644 & 0.739 \\
\hline & Central nucleus & $40.210 \pm 6.246$ & $43.291 \pm 5.649$ & $42.654 \pm 6.794$ & 1.109 & 0.368 & 0.306 & 0.183 & 0.737 \\
\hline & Medial nucleus & $20.219 \pm 5.779$ & $20.390 \pm 3.818$ & $21.452 \pm 3.815$ & 0.811 & 0.450 & 0.658 & 0.396 & 0.215 \\
\hline & Cortical nucleus & $25.228 \pm 3.544$ & $27.040 \pm 3.934$ & $27.368 \pm 3.429$ & 3.288 & $0.045^{\star}$ & 0.324 & 0.014 & 0.127 \\
\hline & CAT & $168.509 \pm 21.032$ & $182.552 \pm 25.746$ & $190.128 \pm 26.655$ & 6.470 & $0.003^{\star \star}$ & 0.123 & $0.001^{\star \star}$ & $0.048^{*}$ \\
\hline & $\begin{array}{l}\text { Paralaminar } \\
\text { nucleus }\end{array}$ & $49.561 \pm 5.853$ & $53.688 \pm 6.877$ & $53.101 \pm 5.820$ & 3.256 & $0.047^{\star}$ & 0.058 & $0.022^{\star}$ & 0.642 \\
\hline \multirow[t]{10}{*}{ Right } & Whole amygdala & $1741.491 \pm 175.730$ & $1856.749 \pm 192.371$ & $1859.253 \pm 168.184$ & 5.184 & $0.009^{\star \star}$ & 0.050 & $0.003^{\star \star}$ & 0.243 \\
\hline & Lateral nucleus & $658.386 \pm 65.822$ & $699.189 \pm 64.786$ & $692.813 \pm 65.799$ & 2.860 & 0.066 & 0.057 & $0.038^{*}$ & 0.814 \\
\hline & Basal nucleus & $448.180 \pm 48.673$ & $478.357 \pm 59.766$ & $478.559 \pm 45.965$ & 3.508 & $0.037^{\star}$ & 0.114 & $0.012^{\star}$ & 0.313 \\
\hline & $\mathrm{ABN}$ & $260.065 \pm 30.681$ & $278.116 \pm 33.518$ & $283.288 \pm 25.640$ & 6.825 & $0.002^{\star \star}$ & 0.115 & $0.001^{\star \star}$ & $0.042^{\star}$ \\
\hline & AAA & $61.595 \pm 6.781$ & $65.147 \pm 7.057$ & $64.180 \pm 6.641$ & 1.151 & 0.324 & 0.168 & 0.25 & 0.851 \\
\hline & Central nucleus & $41.861 \pm 7.571$ & $44.650 \pm 5.929$ & $46.013 \pm 5.886$ & 3.226 & $0.048^{\star}$ & 0.420 & $0.015^{\star}$ & 0.099 \\
\hline & Medial nucleus & $21.761 \pm 4.291$ & $23.858 \pm 5.181$ & $24.000 \pm 4.893$ & 1.203 & 0.308 & 0.386 & 0.130 & 0.504 \\
\hline & Cortical nucleus & $27.501 \pm 2.979$ & $28.732 \pm 3.444$ & $29.276 \pm 3.349$ & 1.730 & 0.187 & 0.560 & 0.072 & 0.222 \\
\hline & CAT & $172.367 \pm 20.692$ & $184.866 \pm 23.368$ & $188.620 \pm 19.389$ & 4.988 & $0.010^{\star}$ & 0.125 & $0.003^{\star \star}$ & 0.115 \\
\hline & $\begin{array}{l}\text { Paralaminar } \\
\text { nucleus }\end{array}$ & $49.776 \pm 5.556$ & $53.834 \pm 7.309$ & $52.502 \pm 5.646$ & 2.520 & 0.090 & 0.065 & 0.057 & 0.911 \\
\hline
\end{tabular}

Data are presented as mean \pm standard deviation. The pairwise comparisons between groups using Bonferroni method. ${ }^{\#} A N C O V A s\left(\right.$ Analysis of covariance). nit: mm ${ }^{3} .{ }^{*} p<0.05$, ${ }^{* *} p<0.01,{ }^{* * *} p<0.001$.

$A B N$, accessory basal nucleus; $A A A$, anterior amygdaloid area; $C A T$, cortico-amygdaloid transition.

affecting threat reactivity and memory (46). The central nucleus is a key output area for expressing innate emotional responses and associated physiological responses, and it connects brainstem controlling specific behaviors and physiological responses. The basal nucleus is another important region of output connecting with the central nucleus; and the striatal areas are related with controlling of instrumental behaviors. In addition, connections from the basal amygdala to the striatum are involved in controlling actions. The lateral nucleus in is believed to tie cortical areas account for processing sensory stimuli with structures responsible for eliciting emotional responses to these stimuli. Therefore, we suggested that the $\mathrm{ABN}$ and CAT may serve as early image markers for differentiating patients with $\mathrm{PBD}$ from $\mathrm{HCs}$ and the volume of basal, lateral, and central nucleus for targeting or tracking the progression of illness in adolescents BD.

The amygdala can be generally partitioned into two major subdivisions: the basolateral (BLA), and centrocorticomedial. The ABN, basal, and lateral nucleus constitute the BLA complex $(25,47)$, which comprises $69 \%$ of the total amygdala volume in humans. The BLA group is thought to represent an integration center for coordinating inputs from certain cortical and subcortical regions, including the prefrontal cortex (PFC), hippocampus, thalamus, and visual cortices; the BLA is involved in learning and memory (48). The cortical, medial, and central nucleus belong to the centrocorticomedial group (49), which has been suggested to receive astrictive information from the medial PFC and BLA, thereby serve as the pathway to generate behavioral, motor, and autonomic emotional responses (50).
The results showed that the decreased volume of amygdala subnuclei in PBD patients were mainly concentrated in the BLA.

In psychiatric disorders, neurocognitive impairments are prevalent and have been associated with poor outcome (51). The cognitive tests used in this study cover a broad range of cognitive abilities, including attentional capacity measured with DST-A and TMT-A; processing speed measured with TMT-A, SCWT-A (color naming), SCWT-B (word reading); working memory/ mental tracking measured with DST-B; visual memory measured with VR I (immediate recall); self-regulation/selfmonitoring measured with SCWT-C (inhibition); and cognitive flexibility measured with TMT-B (Number-Letter Switching) (52). In this study, SCWT, TMT-A, VR I, and DST-B completion scores differed significantly between the patients and HCs. The results provide evidence that manic and euthymic patients with $\mathrm{PBD}$ have significant cognitive impairment, specifically in processing speed, executive function, visual learning, and working memory.

The Spearman correlation of this study indicated that amygdala subnuclei association with VR I scores are primarily in the right BLA (Figures 3B-D). Except for immediate recall memory, VR is also related to visual-perceptual-motor and nonverbal reasoning memory. VR has a widely of clinical and research utility, often employed in $\mathrm{AD}$ (53), posttraumatic stress disorder (54), major depressive Disorder (55), autism spectrum disorder (56). Troster et al. (53) found that VR had excellent sensitivity and specificity in differentiating patients with $\mathrm{AD}$ from HCs. Mak and colleagues (55) found that unipolar and bipolar patients with depression could be distinguished by a 

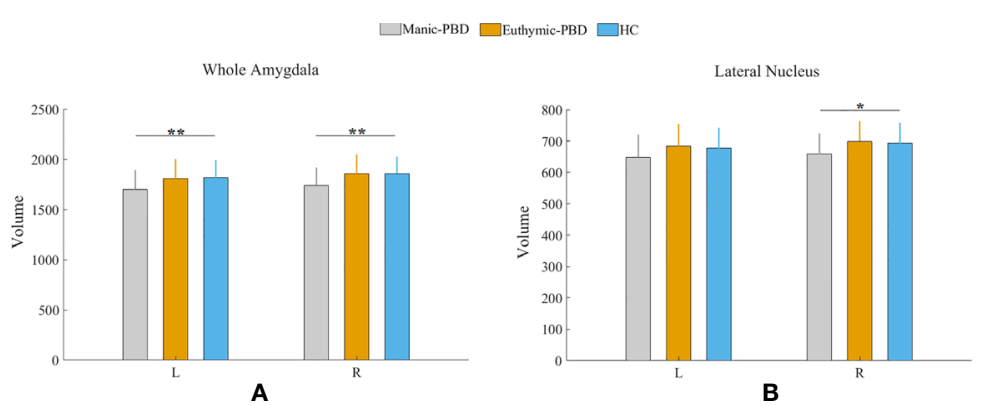

Basal Nucleus

ABN
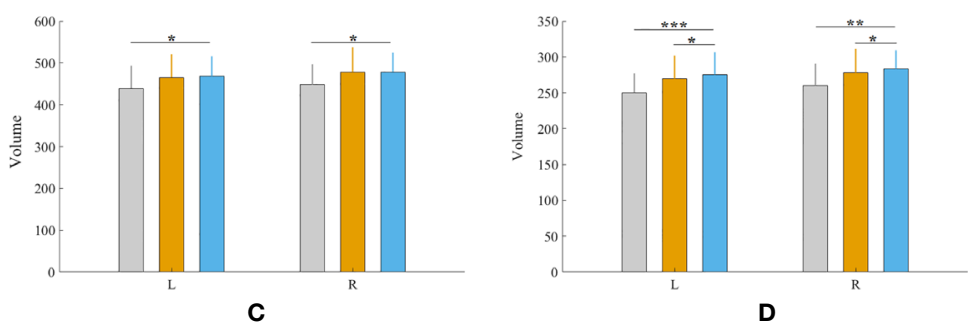

AAA

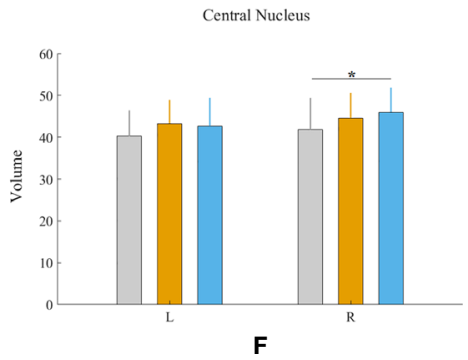

Medial Nucleus

Cortical Nucleus
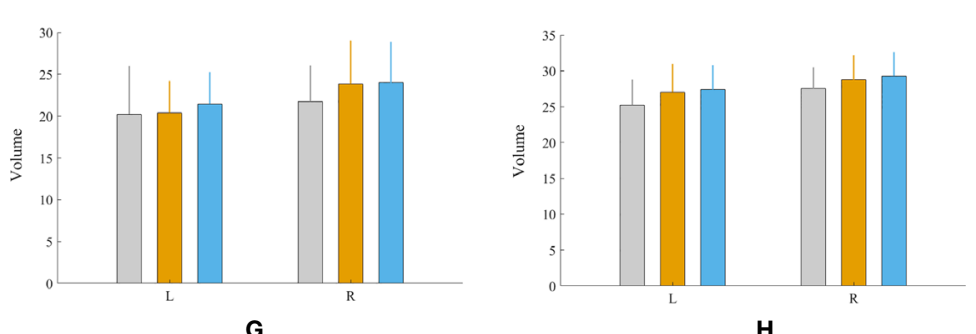

H

CAT

Paralaminar Nucleus
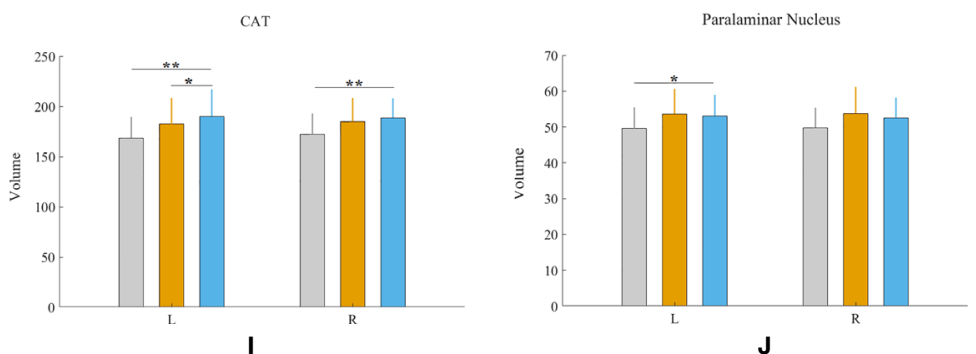

FIGURE 2 | Pairwise comparison of volumes in the manic PBD group, euthymic PBD group, and healthy control group (A,Whole Amygdala volume; B, Lateral Nucleus volume; C, Basal Nucleus volume; D, ABN volume; E, AAA volume; F, Central Nucleus volume; G, Medial Nucleus volume; H, Cortical Nucleus volume; I, CAT volume; J, Paralaminar Nucleus volume). The $Y$-axis represents the mean volume of amygdala and its subnuclei in each group. Unit: $m m^{3}$. ${ }^{\star} p<0.05 ;{ }^{* \star} p<$ 0.01. L, left amygdala; R, right amygdala; ABN, accessory basal nucleus; AAA, anterior amygdaloid area; CAT, cortico-amygdaloid transition. 
A

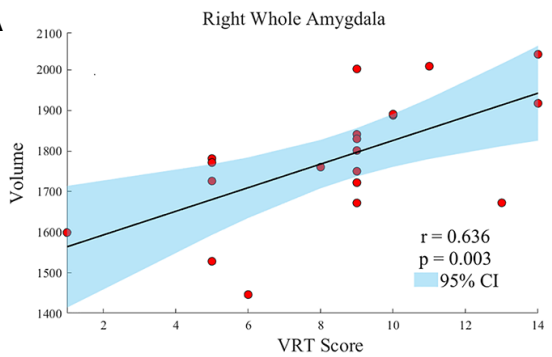

B

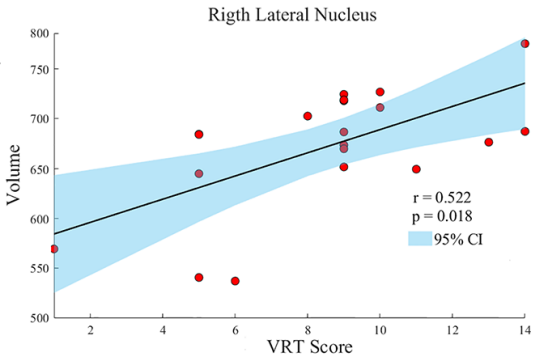

C

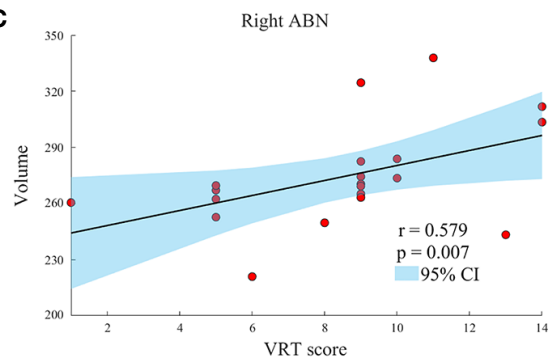

D

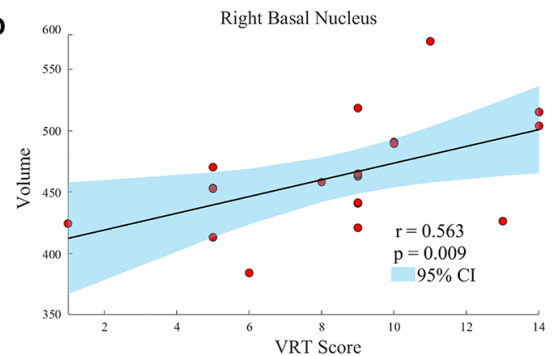

E

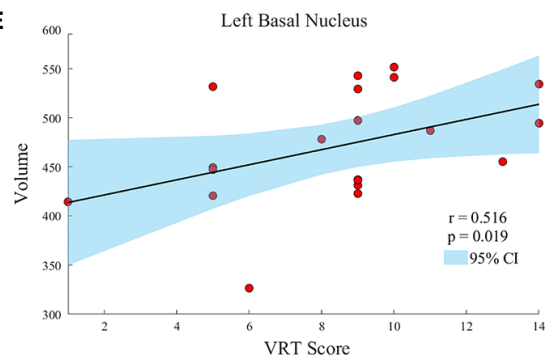

FIGURE 3 | Scatter plots showing the relationships between the amygdala and subnuclei volumes and VR I score in manic patients group (A, Right Whole Amygdala; B, Right Lateral Nucleus; C, Right ABN; D, Right Basal Nucleus; E, Left Basal Nucleus). ABN, accessory basal nucleus; VR I, visual reproduction immediate recall subtest. relatively intact cognitive profile, including TMT and VR. As a result, the right BLA group may serve as early imaging markers for the visual memory dysfunction of manic PBD patients. The relationship between scores in VR I, the right whole amygdala and BLA volumes may evoke a long-existing theory of left-right dissociation of memory systems. This controversial hypothesis suggests that the left amygdala may be responsible for verbal information, whereas visuospatial data may be stored within the right amygdala (57).

There are several limitations in our study. Medication (lithium and other mood stabilizers like valproic acid) could influence the amygdala and subnuclei volumes of patients with PBD. In this study, most of the adolescent patients were taking more than one drug, so we could not rule out drug effects on the results. Moreover, this was a cross-sectional study. Future studies would benefit from longitudinal monitoring to determine whether discrete syndromes have different patterns of amygdala and subnuclei volume changes during an individual's clinical progression.

In conclusion, we used a novel, automated approach to segment and evaluate differences in amygdala and subnuclei volumes in patients with PBD. Together our neuroimaging and cognitive function findings suggest that the volumes of amygdala subnuclei were smaller in manic and euthymic patients with $\mathrm{PBD}$ than that of HCs, especially the ABN and CAT. In addition, visual memory abnormalities might be associated with right whole amygdala, bilateral basal nucleus, right lateral nucleus, and right $\mathrm{ABN}$ volume reductions in patients with manic. Moreover, our findings suggest that smaller BLA group volumes may be an early marker of $\mathrm{PBD}$ progression toward weaker cognitive function.

\section{DATA AVAILABILITY STATEMENT}

All datasets generated for this study are included in the article/ supplementary material.

\section{ETHICS STATEMENT}

The studies involving human participants were reviewed and approved by University of Central South Institutional Review Board. Written informed consent to participate in this study was provided by the participants' legal guardian/next of kin. Written informed consent was obtained from the individual(s), and minor(s)' legal guardian/next of kin, for the publication of any potentially identifiable images or data included in this article.

\section{AUTHOR CONTRIBUTIONS}

JQ, LS, and GL designed the study. WG and LS acquired the data. DC, YG, and WC processed all neuroimaging data. DC and JQ performed all statistical analyses. DC wrote the article, which all 
authors reviewed. All authors contributed to the article and approved the submitted version.

\section{FUNDING}

We are grateful for support from the Fundamental Research Funds for the Central Universities (No. 3332018159 to DC), Funds of the National Natural Science Foundation of China (81371531 to QJ, 81901730 to WC), Key Project of Scientific Research of "12th Five-

\section{REFERENCES}

1. Frias A, Palma C, Farriols N. Comorbidity in pediatric bipolar disorder: prevalence, clinical impact, etiology and treatment. J Affect Disord (2015) 174:378-89. doi: 10.1016/j.jad.2014.12.008

2. Leibenluft E, Charney DS, Pine DS. Researching the pathophysiology of pediatric bipolar disorder. Biol Psychiatry (2003) 53(11):1009-20. doi: 10.1016/S0006-3223(03)00069-6

3. Perlis RH, Miyahara S, Marangell LB, Wisniewski SR, Ostacher M, DelBello $\mathrm{MP}$, et al. Long-term implications of early onset in bipolar disorder: data from the first 1000 participants in the systematic treatment enhancement program for bipolar disorder (STEP-BD). Biol Psychiatry (2004) 55(9):875-81. doi: 10.1016/j.biopsych.2004.01.022

4. Malhi GS, Ivanovski B, Hadzi-Pavlovic D, Mitchell PB, Vieta E, Sachdev P. Neuropsychological deficits and functional impairment in bipolar depression, hypomania and euthymia. Bip Disord (2007) 9(1-2):114-25. doi: 10.1111/ j.1399-5618.2007.00324.x

5. Elias LR, Miskowiak KW, Vale AM, Kohler CA, Kjaerstad HL, Stubbs B, et al. Cognitive Impairment in Euthymic Pediatric Bipolar Disorder: A Systematic Review and Meta-Analysis. J Am Acad Child Adolesc Psychiatry (2017) 56 (4):286-96. doi: 10.1016/j.jaac.2017.01.008

6. Pompili M, Gibiino S, Innamorati M, Serafini G, Del Casale A, De Risio L, et al. Prolactin and thyroid hormone levels are associated with suicide attempts in psychiatric patients. Psychiatry Res (2012) 200(2-3):389-94. doi: 10.1016/j.psychres.2012.05.010

7. Pompili M, Shrivastava A, Serafini G, Innamorati M, Milelli M, Erbuto D, et al. Bereavement after the suicide of a significant other. Indian J Psychiatry (2013) 55(3):256-63. doi: 10.4103/0019-5545.117145

8. Bora E, Pantelis C. Meta-analysis of Cognitive Impairment in First-Episode Bipolar Disorder: Comparison With First-Episode Schizophrenia and Healthy Controls. Schizophr Bull (2015) 41(5):1095-104. doi: 10.1093/ schbul/sbu198

9. Frias A, Palma C, Farriols N. Neurocognitive impairments among youth with pediatric bipolar disorder: a systematic review of neuropsychological research. J Affect Disord (2014) 166:297-306. doi: 10.1016/j.jad.2014.05.025

10. Brambilla P, Hatch JP, Soares JC. Limbic changes identified by imaging in bipolar patients. Curr Psychiatry Rep (2008) 10(6):505-9. doi: 10.1007/ s11920-008-0080-8

11. Sotres-Bayon F, Sierra-Mercado D, Pardilla-Delgado E, Quirk GJ. Gating of fear in prelimbic cortex by hippocampal and amygdala inputs. Neuron. (2012) 76(4):804-12. doi: 10.1016/j.neuron.2012.09.028

12. Mendez-Bertolo C, Moratti S, Toledano R, Lopez-Sosa F, Martinez-Alvarez R, Mah YH, et al. A fast pathway for fear in human amygdala. Nat Neurosci (2016) 19(8):1041-9. doi: 10.1038/nn.4324

13. Asari T, Konishi S, Jimura K, Chikazoe J, Nakamura N, Miyashita Y. Amygdalar enlargement associated with unique perception. Cortex. (2010) 46(1):94-9. doi: 10.1016/j.cortex.2008.08.001

14. Jacobs RH, Renken R, Aleman A, Cornelissen FW. The amygdala, top-down effects, and selective attention to features. Neurosci Biobehav Rev (2012) 36 (9):2069-84. doi: 10.1016/j.neubiorev.2012.05.011

15. Krause-Utz A, Winter D, Schriner F, Chiu CD, Lis S, Spinhoven P, et al. Reduced amygdala reactivity and impaired working memory during dissociation in borderline personality disorder. Eur Arch Psychiatry Clin Neurosci (2018) 268(4):401-15. doi: 10.1007/s00406-017-0806-x
Year Plan" in Medical Research of the Army (BWS11J063 to GL), Academic Promotion Programme of Shandong First Medical University (No. 2019QL009), and JQ was supported by the Taishan Scholars Program of Shandong Province (No. TS201712065).

\section{ACKNOWLEDGMENTS}

We thank LetPub (www.letpub.com) for its linguistic assistance during the preparation of this manuscript.

16. Kryza-Lacombe M, Brotman MA, Reynolds RC, Towbin K, Pine DS, Leibenluft E, et al. Neural mechanisms of face emotion processing in youths and adults with bipolar disorder. Bip Disord (2019) 21(4):309-20. doi: 10.1111/bdi.12768

17. Kalmar JH, Wang F, Chepenik LG, Womer FY, Jones MM, Pittman B, et al. Relation between amygdala structure and function in adolescents with bipolar disorder. J Am Acad Child Adolesc Psychiatry (2009) 48(6):636-42. doi: 10.1097/CHI.0b013e31819f6fbc

18. Kelley R, Chang KD, Garrett A, Alegria D, Thompson P, Howe M, et al. Deformations of amygdala morphology in familial pediatric bipolar disorder. Bip Disord (2013) 15(7):795-802. doi: 10.1111/bdi.12114

19. Blumberg HP, Kaufman J, Martin A, Whiteman R, Zhang JH, Gore JC, et al. Amygdala and hippocampal volumes in adolescents and adults with bipolar disorder. Arch Gen Psychiatry (2003) 60(12):1201-8. doi: 10.1001/ archpsyc.60.12.1201

20. DelBello MP, Zimmerman ME, Mills NP, Getz GE, Strakowski SM. Magnetic resonance imaging analysis of amygdala and other subcortical brain regions in adolescents with bipolar disorder. Bip Disord (2004) 6(1):43-52. doi: 10.1046/ j.1399-5618.2003.00087.x

21. Pfeifer JC, Welge J, Strakowski SM, Adler CM, DelBello MP. Meta-analysis of amygdala volumes in children and adolescents with bipolar disorder. $J \mathrm{Am}$ Acad Child Adolesc Psychiatry (2008) 47(11):1289-98. doi: 10.1097/ CHI.0b013e318185d299

22. Strakowski SM, Delbello MP, Adler CM. The functional neuroanatomy of bipolar disorder: a review of neuroimaging findings. Mol Psychiatry (2005) 10 (1):105-16. doi: 10.1038/sj.mp.4001585

23. Swayze V, Andreasen NC, Alliger RJ, Ehrhardt JC, Yuh WT. Structural brain abnormalities in bipolar affective disorder. Ventricular enlargement and focal signal hyperintensities. Arch Gen Psychiatry (1990) 47(11):1054-9. doi: 10.1001/archpsyc. 1990.01810230070011

24. Killgore WD, Rosso IM, Gruber SA, Yurgelun-Todd DA. Amygdala volume and verbal memory performance in schizophrenia and bipolar disorder. Cognit Behav Neurol (2009) 22(1):28-37. doi: 10.1097/WNN. 0b013e318192cc67

25. Aghamohammadi-Sereshki A, Hrybouski S, Travis S, Huang Y, Olsen F, Carter R, et al. Amygdala subnuclei and healthy cognitive aging. Hum Brain Mapp (2019) 40(1):34-52. doi: 10.1002/hbm.24353

26. Usher J, Leucht S, Falkai P, Scherk H. Correlation between amygdala volume and age in bipolar disorder - a systematic review and meta-analysis of structural MRI studies. Psychiatry Res (2010) 182(1):1-8. doi: 10.1016/ j.pscychresns.2009.09.004

27. Hibar DP, Westlye LT, van Erp TG, Rasmussen J, Leonardo CD, Faskowitz J, et al. Subcortical volumetric abnormalities in bipolar disorder. Mol Psychiatry (2016) 21(12):1710-6. doi: 10.1038/mp.2015.227

28. Wegbreit E, Cushman GK, Puzia ME, Weissman AB, Kim KL, Laird AR, et al. Developmental meta-analyses of the functional neural correlates of bipolar disorder. JAMA Psychiatry (2014) 71(8):926-35. doi: 10.1001/jamapsychiatry. 2014.660

29. Ding SL, Royall JJ, Sunkin SM, Ng L, Facer BA, Lesnar P, et al. Comprehensive cellular-resolution atlas of the adult human brain. J Comp Neurol (2017) 525 (2):407. doi: 10.1002/cne.24130

30. Kalin NH, Shelton SE, Davidson RJ. The role of the central nucleus of the amygdala in mediating fear and anxiety in the primate. J Neurosci (2004) 24 (24):5506-15. doi: 10.1523/JNEUROSCI.0292-04.2004 
31. Dong X, Li S, Kirouac GJ. Collateralization of projections from the paraventricular nucleus of the thalamus to the nucleus accumbens, bed nucleus of the stria terminalis, and central nucleus of the amygdala. Brain Struct Funct (2017) 222(9):3927-43. doi: 10.1007/s00429-017-1445-8

32. Saygin ZM, Kliemann D, Iglesias JE, van der Kouwe AJW, Boyd E, Reuter M, et al. High-resolution magnetic resonance imaging reveals nuclei of the human amygdala: manual segmentation to automatic atlas. Neuroimage (2017) 155:370-82. doi: 10.1016/j.neuroimage.2017.04.046

33. Association AP. Diagnostic and Statistical Manual of Psychiatric Disorders, fifth ed. Arlington, MA: American Psychiatric Publishing (2013).

34. Traci W, Olivier E, Mark Mahone Lisa A. Jacobson. Wechsler Intelligence Scale for Children. Can J Sch Psychol (2018) 19(Suppl 1):221-34. doi: 10.1007/ 978-3-319-56782-2_1605-2

35. Kaufman J, Birmaher B, Brent D, Rao U, Flynn C, Moreci P, et al. Schedule for Affective Disorders and Schizophrenia for School-Age Children-Present and Lifetime Version (K-SADS-PL): initial reliability and validity data. J Am Acad Child Adolesc Psychiatry (1997) 36(7):980-8. doi: 10.1097/00004583199707000-00021

36. Geller B, Zimerman B, Williams M, Bolhofner K, Craney JL, DelBello MP, et al. Reliability of the Washington University in St. Louis Kiddie Schedule for Affective Disorders and Schizophrenia (WASH-U-KSADS) mania and rapid cycling sections. J Am Acad Child Adolesc Psychiatry (2001) 40(4):450-5. doi: 10.1097/00004583-200104000-00014

37. Wood A, Kroll L, Moore A, Harrington R. Properties of the mood and feelings questionnaire in adolescent psychiatric outpatients: a research note. J Child Psychol Psychiatry (1995) 36(2):327-34. doi: 10.1111/j.1469-7610.1995.tb01828.x

38. Young RC, Biggs JT, Ziegler VE, Meyer DA. A rating scale for mania: reliability, validity and sensitivity. Br J Psychiatry (1978) 133:429-35. doi: 10.1192/bjp.133.5.429

39. Moran L, Yeates KO. Stroop Color and Word Test, Children's Version. Encyclopedia of Clinical Neuropsychology. New York, NY: Springer (2011).

40. Ralph M, Reitan, Deborah, Wolfson. Category test and trail making test as measures of frontal lobe functions. Clin Neuropsychol (1995) 9:50-6. doi: $10.1080 / 13854049508402057$

41. Welch LW, Nimmerrichter A, Gilliland R, King DE, Martin PR. "Wineglass" Confabulations Among Brain- Damaged Alcoholics on the Wechsler Memory Scale-Revised Visual Reproduction Subtest. Cortex. (1997) 33(3):0-551. doi: 10.1016/S0010-9452(08)70235-1

42. Eggins PS, Hatton SN, Hermens DF, Hickie IB, Lagopoulos J. Subcortical volumetric differences between clinical stages of young people with affective and psychotic disorders. Psychiatry Res Neuroimag (2018) 271:8-16. doi: 10.1016/j.pscychresns.2017.11.015

43. Markesbery WR. Neuropathologic alterations in mild cognitive impairment: a review. J Alzheimers Dis (2010) 19(1):221-8. doi: 10.3233/JAD-2010-1220

44. McGaugh JL. Memory-a century of consolidation. Science (2000) 287 (5451):248-51. doi: 10.1126/science.287.5451.248

45. Trobe DJ. The Human Brain. An Introduction to Its Functional Anatomy, 6th Edition. J Neuro-Ophthalmol (2010) 30(1):107. doi: 10.1097/01.wno. 0000369168.32606 .54
46. Brown SSG, Rutland JW, Verma G, Feldman RE, Schneider M, Delman BN, et al. Ultra-High-Resolution Imaging of Amygdala Subnuclei Structural Connectivity in Major Depressive Disorder. Biol Psychiatry Cognit Neurosci Neuroimaging (2020) 5(2):184-93. doi: 10.1016/j.bpsc.2019.07.010

47. LeDoux J. The amygdala. Curr Biol (2007) 17(20):R868-74. doi: 10.1016/ j.cub.2007.08.005

48. Roozendaal B, McEwen BS, Chattarji S. Stress, memory and the amygdala. Nat Rev Neurosci (2009) 10(6):423-33. doi: 10.1038/nrn2651

49. Aggleton J. The amygdala: a functional analysis. New York, NY: Oxford University Press (2000).

50. Pessoa L. Reprint of: Emotion and cognition and the amygdala: from "what is it?" to "what's to be done"?. Neuropsychologia (2011) 49(4):681-94. doi: 10.1016/j.neuropsychologia.2011.02.030

51. Joseph MF, Frazier TW, Youngstrom EA, Soares JC. A quantitative and qualitative review of neurocognitive performance in pediatric bipolar disorder. J Child Adolesc Psychopharmacol (2008) 18(6):595-605. doi: 10.1089/cap.2008.064

52. Sparding T, Silander K, Palsson E, Ostlind J, Ekman CJ, Sellgren CM, et al. Classification of cognitive performance in bipolar disorder. Cognit Neuropsychiatry (2017) 22(5):407-21. doi: 10.1080/13546805.2017.1361391

53. Troster AI, Butters N, Salmon DP, Cullum CM, Jacobs D, Brandt J, et al. The diagnostic utility of savings scores: differentiating Alzheimer's and Huntington's diseases with the logical memory and visual reproduction tests. J Clin Exp Neuropsychol (1993) 15(5):773-88. doi: 10.1080/ 01688639308402595

54. Vasterling JJ, Aslan M, Lee LO, Proctor SP, Ko J, Jacob S, et al. Longitudinal Associations among Posttraumatic Stress Disorder Symptoms, Traumatic Brain Injury, and Neurocognitive Functioning in Army Soldiers Deployed to the Iraq War. J Int Neuropsychol Soc (2018) 24(4):311-23. doi: 10.1017/ S1355617717001059

55. Mak ADP, Lau DTY, Chan AKW, So SHW, Leung O, Wong SLY, et al. Cognitive Impairment In Treatment-Naive Bipolar II and Unipolar Depression. Sci Rep (2018) 8(1):1905. doi: 10.1038/s41598-018-20295-3

56. Funabiki Y, Shiwa T. Weakness of visual working memory in autism. Autism Res (2018) 11(9):1245-52. doi: 10.1002/aur.1981

57. Markowitsch HJ. Differential contribution of right and left amygdala to affective information processing. Behav Neurol (1998) 11(4):233-44. doi: $10.1155 / 1999 / 180434$

Conflict of Interest: The authors declare that the research was conducted in the absence of any commercial or financial relationships that could be construed as a potential conflict of interest.

Copyright $\odot 2020 \mathrm{Cui}$, Guo, Cao, Gao, Qiu, Su, Jiao and Lu. This is an open-access article distributed under the terms of the Creative Commons Attribution License (CC BY). The use, distribution or reproduction in other forums is permitted, provided the original author(s) and the copyright owner(s) are credited and that the original publication in this journal is cited, in accordance with accepted academic practice. No use, distribution or reproduction is permitted which does not comply with these terms. 